\title{
ON THE LOCATION OF THE ZEROS OF CERTAIN ORTHOGONAL FUNCTIONS ${ }^{1}$
}

\section{J. L. WALSH AND J. P. EVANS}

Let $R$ be a finite region of the z-plane and let $L^{2}(R)$ denote the class of functions $f(z)$ each analytic in $R$ with $\iint_{R}|f(z)|^{2} d S<\infty$. Let the points $\beta_{1}, \beta_{2}, \beta_{3}, \cdots$ be given interior to $R$ and let $\phi_{n}(z)$ be that function of class $L^{2}(R)$ for which $\phi_{n}\left(\beta_{1}\right)=\phi_{n}\left(\beta_{2}\right)=\cdots=\phi_{n}\left(\beta_{n-1}\right)=0$, $\phi_{n}\left(\beta_{n}\right)=1$, and which minimizes $\iint_{R}\left|\phi_{n}(z)\right|^{2} d S$ over the class $L^{2}(R)$. If the points $\beta_{1}, \beta_{2}, \beta_{3}, \cdots$ are not all distinct these requirements of interpolation on $\phi_{n}(z)$ are to be interpreted in the usual way in the theory of interpolation, to refer to the vanishing of suitable derivatives of $\phi_{n}(z)$ in multiple points $\beta_{k}$.

The purpose of this note is to establish results on the location of zeros of the functions $\phi_{n}(z)$. Our main result is the

THEOREM. Let $R$ be a finite region of the z-plane which contains in its interior the points $\beta_{1}, \beta_{2}, \beta_{3}, \cdots$ and all limit points of the $\beta_{n}$. Then any circle which together with its interior lies in $R$ and which contains in its interior all limit points of the $\beta_{n}$, contains in its interior no zero of $\phi_{n}(z)$ (other than $\beta_{k}, k=1, \cdots, n-1$ ) for $n$ sufficiently large.

The functions $\phi_{n}(z)$ were first introduced by Bergman [1] in the case that $\beta_{n}$ is independent of $n$, and were later studied by Walsh and Davis [6] in the case that the $\beta_{n}$ approach a limit. The totality of zeros of such functions were first studied by the present writers [2] in the analogous case that the double integral over $R$ is replaced by a line integral over the boundary of $R$; our theorem and other results on the totality of zeros of the $\phi_{n}(z)$ are of particular significance with reference to the asymptotic properties of the functions, and to the divergence of series $\sum_{1}^{\infty} a_{n} \phi_{n}(z)$.

The existence of the functions $\phi_{n}(z)$ follows readily from the theory of normal families, and uniqueness is also easily proved [2]. We introduce the normalized functions $\phi_{n}^{*}(z) \equiv \phi_{n}(z) /\left[\iint_{R}\left|\phi_{n}(z)\right|^{2} d S\right]^{1 / 2}$.

$A$ rapid sketch of the ideas underlying the proof is as follows. The functions $\phi_{n}^{*}(z)$ are bounded in norm in $R$, hence uniformly bounded in absolute value on any closed set interior to $R$. The fact that $\phi_{n}^{*}(z)$ has $n-1$ zeros in $R$ but not near the boundary of $R$ implies that

Received by the editors February 6, 1956.

1 This research was sponsored in part by the U. S. Air Force, Office of Scientific Research of the Air Research and Development Command. 
$\phi_{n}^{*}(z) \rightarrow 0$ uniformly on any closed set in $R$. Thus the subset of $R$ on which $\left|\phi_{n}^{*}(z)\right|$ is not small lies near the boundary of $R$, and the situation is similar to that in which the given functions are defined in terms of a line integral over the boundary of $R$.

As a first step in the formal proof, we establish the

LEMma. Let $\phi(z)$ be analytic and of modulus not exceeding the constant $L$ for $|z| \leqq A$ and let $\phi\left(\beta_{k}\right)=0, k=n_{0}, n_{0}+1, \cdots, n-1$, with $\left|\beta_{k}\right| \leqq A \rho, \rho<1$. Then in $|z| \leqq|Z|<A$ we have

$$
|\phi(z)| \leqq L\left(\frac{|Z / A|+\rho}{1+\rho|Z / A|}\right)^{n-1-n_{0}} .
$$

If $A=1$ we have by the maximum principle

$$
|\phi(z)| / \prod_{k=n_{0}}^{n-1}\left|\frac{z-\beta_{n}}{1-\bar{\beta}_{n} z}\right| \leqq L \quad \text { for }|z| \leqq 1,
$$

and since $[3$, p. 290]

$$
\left|\frac{z-\beta_{k}}{1-\bar{\beta}_{k} z}\right| \leqq \frac{|Z|+\rho}{1+\rho|Z|}<1, \text { for }|z| \leqq|Z|<1,
$$

the result follows for this case. The conclusion of the lemma for arbitrary $A$ follows immediately by making the transformation $\boldsymbol{z}^{\prime}=\boldsymbol{z} / \boldsymbol{A}$.

We now turn to the proof of the theorem. If $S_{1}$ and $S_{2}$ are any two point sets, $d\left(S_{1}, S_{2}\right)$ shall denote the distance from $S_{1}$ to $S_{2}$. The closure of $S_{1}$ is denoted by $\bar{S}_{1}$. Let $T$ be any circle (i.e. circumference) which together with its interior $D$ lies in $R$ such that the points $\beta_{k}, k \geqq n_{0}$, lie in $D$. Since $d(T, C)>0$, where $C$ is the boundary of $R$, there exists a constant $d$ such that $d\left(\beta_{n}, T\right) \geqq d>0$ when $n \geqq n_{0}$. We now choose a circle $T_{1}$ contained in $R$ and containing $T$ in its interior; we consider the integral $\iint_{R-\bar{D}_{1}}\left|\phi_{n}^{*}(z)\right|^{2}(z-\alpha)^{-1} d S$, where $D_{1}$ is the interior of $T_{1}$ and $\alpha$ is an arbitrary point in $D$. We can interpret the conjugate of this integral as the force at $z=\alpha$ due to a spread of nonnegative matter over $R-\bar{D}_{1}$ which repels according to the law of inverse distance. Since the set $R-\bar{D}_{1}$ lies exterior to $T$ and $\alpha$ is interior to $T$, this force is equal to the force at $\alpha$ due to the same total mass concentrated at a suitable point $\beta_{n}^{\prime}$ exterior to $T[4, \mathrm{pp} .13$, 247]:

(1) $\iint_{R-\bar{D}_{1}}\left|\phi_{n}^{*}(z)\right|^{2} \frac{d S}{z-\alpha}=\iint_{R_{1}-\bar{D}_{1}}\left|\phi_{n}^{*}(z)\right|^{2} d S /\left(\beta_{n}^{\prime}-\alpha\right)$. 
We shall now show that the assumption $\phi_{n}^{*}(\alpha)=0 \quad\left(\alpha \neq \beta_{k}\right.$, for $k=1,2, \cdots, n)$ implies that the point $\beta_{n}^{\prime}$ cannot lie exterior to $T$ when $n$ is sufficiently large, and we are thus led to a contradiction. If $\phi_{n}^{*}(\alpha)=0$ the function $\left(z-\beta_{n}\right) \phi_{n}^{*}(z) /(z-\alpha)$ when suitably defined at $z=\alpha$ is of class $L^{2}(R)$ and vanishes in the points $\beta_{1}, \beta_{2}, \cdots, \beta_{n}$ and hence (compare [5]) is orthogonal to $\phi_{n}^{*}(z)$. That is to say,

$$
\begin{gathered}
\iint_{R} \frac{z-\beta_{n}}{z-\alpha}\left|\phi_{n}^{*}(z)\right|^{2} d S=0 \\
\iint_{R}\left|\phi_{n}^{*}(z)\right|^{2} \frac{d S}{z-\alpha}=\iint_{R}\left|\phi_{n}^{*}(z)\right|^{2} d S /\left(\beta_{n}-\alpha\right)=\frac{1}{\beta_{n}-\alpha} .
\end{gathered}
$$

We next show that as a consequence of (1), (2), and the lemma we have for given $\eta(>0)$ and for all $n$ sufficiently large

$$
\begin{aligned}
&\left|\frac{1}{\beta_{n}{ }^{\prime}-\alpha}\right|<\frac{2}{d\left(T, T_{1}\right)}, \\
&\left|\frac{1}{\beta_{n}-\alpha}-\frac{1}{\beta_{n}^{\prime}-\alpha}\right|<\eta .
\end{aligned}
$$

Let $T_{2}$ be a circle concentric with $T_{1}$ which together with its interior lies in $R$ and which contains $T_{1}$ in its interior. Since $\iint_{R}\left|\phi_{n}^{*}(z)\right|^{2} d S=1$ there exists $\left[3\right.$, p. 96] a constant $L$ depending on $T_{2}$ but not on $\phi_{n}^{*}(z)$ such that $\left|\phi_{n}^{*}(z)\right| \leqq L$ for $z$ on and interior to $T_{2}$. We let $T_{2}$ play the rôle of the circle $|z|=A$ in the lemma. It then follows from the lemma that when $n>n_{0}+1$ there exists a positive constant $r(<1)$ independent of $n$ and of $z$ on $\bar{D}_{1}$ such that

$$
\left|\phi_{n}^{*}(z)\right| \leqq L r^{n-1-n_{0}}, \quad z \text { on } \bar{D}_{1} .
$$

The finite region $R$ is contained in a circle of radius $M$ and by (5) there exists an $n_{1}\left(>n_{0}+1\right)$ such that when $n \geqq n_{1}$ we have $\left|\phi_{n}^{*}(z)\right|^{2}$ $<1 / 2 \pi M^{2}, z$ in $\bar{D}_{1}$. Thus when $n \geqq n_{1}$ we have

$$
\begin{aligned}
\left.\left|\iint_{R-\bar{D}_{1}}\right| \phi_{n}^{*}(z)\right|^{2} d S-1 \mid & =\left.\left|\iint_{R-\bar{D}_{1}}\right| \phi_{n}^{*}(z)\right|^{2} d S-\iint_{R}\left|\phi_{n}^{*}(z)\right|^{2} d S \mid \\
& =\left.\left|\iint_{D_{1}}\right| \phi_{n}^{*}(z)\right|^{2} d S \mid<\frac{1}{2},
\end{aligned}
$$

or

$$
\left.\left|\iint_{R-\bar{D}_{1}}\right| \phi_{n}^{*}(z)\right|^{2} d S \mid>\frac{1}{2} \quad \text { when } n \geqq n_{1} \text {. }
$$


Also

$$
\left.\left.\left|\iint_{R-\bar{D}_{1}}\right| \phi_{n}^{*}(z)\right|^{2} \frac{d S}{z-\alpha}\left|\leqq \frac{1}{d\left(T, T_{1}\right)} \iint_{R}\right| \phi_{n}^{*}(z)\right|^{2} d S=\frac{1}{d\left(T, T_{1}\right)}
$$

for all $n$ and all $\alpha$ interior to $T$. Hence, from this last inequality and from (1)

$$
\begin{aligned}
\left|\frac{1}{\beta_{n}{ }^{\prime}-\alpha}\right| & =\left|\iint_{R-\bar{D}_{1}}\left[\left|\phi_{n}^{*}(z)\right|^{2} /(z-\alpha)\right] d S\right| /\left.\left|\iint_{R-\bar{D}_{1}}\right| \phi_{n}^{*}(z)\right|^{2} d S \\
& <\frac{2}{d\left(T, T_{1}\right)}, \quad n \geqq n_{1},
\end{aligned}
$$

and (3) is established.

By virtue of (5) there exists an $n_{2}\left(>n_{0}+1\right)$ such that when $n \geqq n_{2}$

$$
\left|\phi_{n}^{*}(z)\right|^{2}<\frac{\eta d\left(T, T_{1}\right)}{4 \pi M^{2}}, \quad \quad z \text { in } \bar{D}_{1} \text {. }
$$

Thus, for $z$ on $T_{1}$ and hence for $z$ in $D_{1}$ we also have $\left(n \geqq n_{2}\right)$ by (6)

$$
\frac{\left|\phi_{n}^{*}(z)\right|^{2}}{|z-\alpha|}<\frac{\eta}{4 \pi M^{2}}
$$

since the function $\phi_{n}^{*}(z) /(z-\alpha)$ is analytic in $\bar{D}_{1}$ (when suitably defined for $z=\alpha)$. Then, by (6) for $n \geqq n_{2}$

$$
\begin{aligned}
\left.\left|1-\iint_{R-\bar{D}_{1}}\right| \phi_{n}^{*}(z)\right|^{2} d S \mid & =\left.\left|\iint_{R}\right| \phi_{n}^{*}(z)\right|^{2} d S-\iint_{R-\bar{D}_{1}}\left|\phi_{n}^{*}(z)\right|^{2} d S \mid \\
& =\left.\left|\iint_{D_{1}}\right| \phi_{n}(z)\right|^{2} d S \mid<\frac{\eta d\left(T, T_{1}\right)}{4},
\end{aligned}
$$

and by (7) for $n \geqq n_{2}$

$$
\begin{aligned}
\left|\frac{1}{\beta_{n}-\alpha}-\iint_{R-\bar{D}_{1}}\right| \phi_{n}^{*}(z)\left|2 \frac{d S}{z-\alpha}\right| \\
=\left.\left|\iint_{R}\right| \phi_{n}^{*}(z)\right|^{2} \frac{d S}{z-\alpha}-\iint_{R-\bar{D}_{1}}\left|\phi_{n}^{*}(z)\right| 2 \frac{d S}{z-\alpha} \mid \\
=\left.\left|\iint_{D_{1}}\right| \phi_{n}^{*}(z)\right|^{2} \frac{d S}{z-\alpha} \mid<\frac{n}{4} .
\end{aligned}
$$

Hence, when $n \geqq \max \left(n_{1}, n_{2}\right)$ we have 


$$
\begin{aligned}
\left|\frac{1}{\beta_{n}-\alpha}-\frac{1}{\beta_{n}^{\prime}-\alpha}\right|= & \mid\left[\frac{1}{\beta_{n}-\alpha}-\frac{1}{\beta_{n}^{\prime}-\alpha} \iint_{R-\bar{D}_{1}}\left|\phi_{n}^{*}(z)\right|^{2} d S\right] \\
& -\frac{1}{\beta_{n}^{\prime}-\alpha}\left[1-\iint_{R-\bar{D}_{1}}\left|\phi_{n}^{*}(z)\right|^{2} d S\right] \mid \\
& <\frac{\eta}{4}+\frac{\eta d\left(T, T^{\prime}\right)}{4} \cdot \frac{2}{d\left(T, T^{\prime}\right)}<\eta,
\end{aligned}
$$

and (4) is established.

We are now in a position to show that $\beta_{n}{ }^{\prime}$ cannot lie exterior to $T$. If in (4) we set $\eta=1 / d\left(T, T_{1}\right)$, it follows from (3) and (4) that for $n$ sufficiently large (and independent of $\alpha$ in $D$ ) we have $1 /\left|\beta_{n}-\alpha\right|$ $<3 / d\left(T, T_{1}\right)$. Since $1 / 2 M<1 /\left|\beta_{n}-\alpha\right|$ for all $n$ sufficiently large and for all $\alpha$ in $D$, we have by setting $\eta=1 / 4 M$ in (4) that $1 / 4 M$ $<1 /\left|\beta_{n}^{\prime}-\alpha\right|$ for $n$ sufficiently large and for all $\alpha$ in $D$. Thus for all $n$ sufficiently large we have

$$
\frac{1}{4 M}<\frac{1}{\left|\beta_{n}-\alpha\right|}<\frac{3}{d\left(T, T_{1}\right)}, \quad \frac{1}{4 M}<\frac{1}{\left|\beta_{n}^{\prime}-\alpha\right|}<\frac{3}{d\left(T, T_{1}\right)} .
$$

Since the function $f(Z) \equiv 1 / Z$ is uniformly continuous on any closed bounded set $B$ of the $Z$-plane not containing $Z=0$, there corresponds to arbitrary $\epsilon(>0)$ a $\delta(>0)$ such that for all $Z_{1}$ and $Z_{2}$ on $B$ with $\left|Z_{1}-Z_{2}\right|<\delta$ we have $\left|1 / Z_{1}-1 / Z_{2}\right|<\epsilon$. We now choose $B$ as the set $1 / 4 M \leqq|Z| \leqq 3 / d\left(T, T_{1}\right)$ with $\epsilon=d \leqq d\left(\beta_{n}, T\right)$. In (4) we set $\eta=\delta$, whence it follows that there exists an $n_{3}$ such that when $n \geqq n_{3}$ we have $\left|1 /\left(\beta_{n}-\alpha\right)-1 /\left(\beta_{n}^{\prime}-\alpha\right)\right|<\delta$ and such that (8) is valid. Then with $Z_{1}=1 /\left(\beta_{n}-\alpha\right), Z_{2}=1 /\left(\beta_{n}^{\prime}-\alpha\right)$ when $n \geqq n_{3}$ we have $\mid\left(\beta_{n}-\alpha\right)$ $-\left(\beta_{n}^{\prime}-\alpha\right)|=| \beta_{n}-\beta_{n}^{\prime} \mid<d \leqq d\left(\beta_{n}, T\right)$ and hence the points $\beta_{n}^{\prime}$ cannot lie exterior to $T$ when $n \geqq n_{3}$. Since the choice of $n_{3}$ is independent of $\alpha$ interior to $T$, this contradiction completes the proof of the theorem.

The application of this theorem in the study of asymptotic properties of the $\phi_{n}^{*}(z)$ and in the study of divergence of series $\sum_{1}^{\infty} a_{n} \phi_{n}^{*}(z)$ is wholly analogous to the treatment previously given [2] and is left to the reader.

\section{BIBLIOGRAPHY}

1. S. Bergman, The kernel function and conformal mapping, Mathematical Surveys, no. 5, American Mathematical Society, 1950.

2. J. P. Evans and J. L. Walsh, On interpolation to a given analytic function by analytic functions of minimum norm, Trans. Amer. Math. Soc. vol. 79 (1955) pp. 158172. 
3. J. L. Walsh, Interpolation and approximation by rational functions in the complex domain, Amer. Math. Soc. Colloquium Publications, vol. 20, 1935.

4. - The location of critical points of analytic and harmonic functions, Amer. Math. Soc. Colloquium Publications, vol. 34, 1950.

5. - An interpolation problem for harmonic functions, Amer. J. Math. vol. 76 (1954) pp. 259-272. Also Determination d'une fonction analytique par ses valeurs données dans une infinité denombrable de points, Bull. Soc. Math. de Belgique (1955) pp. 52-70.

6. J. L. Walsh and Philip Davis, Interpolation and orthonormal systems, Journal d'Analyse Mathématique vol. 2 (1952) pp. 1-28.

HARVARD UNIVERSITY AND

Smith College

\title{
PARTIALLY BOUNDED CONTINUED FRACTIONS
}

\author{
H. S. WALL
}

For each complex number sequence $a, f(a)$ denotes the continued fraction

$$
\frac{1}{1}+\frac{a_{1}}{1}+\frac{a_{2}}{1}+\frac{a_{3}}{1}+\cdots
$$

The statement that $f(a)$ is partially bounded ${ }^{1}$ means that the sequence $a$ has a bounded infinite subsequence. If $f(a)$ is partially bounded, the series $\sum\left|b_{p}\right|$ diverges, where $b_{1}=1, a_{p}=1 / b_{p} b_{p+1}, p=1,2, \cdots,-\mathrm{a}$ necessary condition for convergence of $f(a)$.

Any continued fraction $f(a)$ such that $\sum\left|b_{p}\right|$ diverges is convergent provided its even and odd parts are absolutely convergent, ${ }^{2}$ i.e. provided the series $\sum\left|f_{2 p+2}-f_{2 p}\right|$ and $\sum\left|f_{2 p+1}-f_{2 p-1}\right|$ are convergent, where $\left\{f_{p}\right\}_{p=1}^{\infty}$ is the sequence of approximants. The simple convergence of the even and odd parts of $f(a)$, together with the divergence of $\sum\left|b_{p}\right|$, is not sufficient for the convergence of $f(a)$, (Theorem 3). However, the simple convergence of the even and odd parts of the partially bounded continued fraction $f(a)$ is sufficient for the convergence of $f(a)$. In fact, we have this theorem:

TheOREM 1. Suppose there is a positive integer $k$ such that the sub-

Presented to the Society, December 27, 1955; received by the editors October 27, 1955 and, in revised form, December 27, 1955.

${ }^{1} f(a)$ is called bounded if the sequence $a$ is bounded - a condition equivalent to the boundedness of a certain infinite matrix. Cf. H. S. Wall, Analytic theory of continued fractions, 1948, p. 110. (Referred to later on as AT.)

2 Trans. Amer. Math. Soc. vol. 67 (1949) pp. 368-380. 